\title{
JOINT ENTERPRISE
}

Tissue engineering is as much a mechanical as a biological problem. Although the field's focus has been largely on getting cells to grow in vitro in the right morphology - a question increasingly now concerned with eliciting the requisite modes of stem-cell differentiation through appropriate environmental cues there are many applications in which an engineered tissue's dynamic properties are at least as important as the static ones. That's why tissues associated with moving parts of the body, such as the trachea, remain among the hardest to 'grow' in a medically viable form.

It is precisely because the mechanical performance of some biological tissues is so admirable that they present such a challenge. Of these, cartilage - the connective tissue at the ends of long bones - is among the most impressive. It can provide lubrication and joint articulation without compromising wear or damage for seven or more decades of constant use. Of course, the problem is that it does degenerate eventually, and the resulting osteoarthritis is a source of pain for millions of people. Although the static mechanical properties of native cartilage have been reasonably well matched in artificial tissues $^{1}$, it is harder to replicate the lubricating function ${ }^{2}$.

An alternative to growing real cartilage with the right mechanics is to mimic its properties in a biocompatible material. Indeed, there is plenty worth emulating here in a synthetic material that might find tribological applications beyond medicine. It has been long recognized that cartilage, like so many biological tissues, has a hierarchical structure ${ }^{3}$; the question is how much of, or how closely, this structure need be copied to achieve acceptable mimicry of function.

Previous efforts to make wholly synthetic cartilage have tended to fixate on just one aspect of its behaviour $^{4}$ - not because that is all that's needed, but because it was all that's feasible. But the tribological superiority of natural cartilage relies on a synergy that will only be attainable by approaching it as an integrated system.

Greene et al. have now taken a step towards a more sophisticated cartilage mimic that combines two of the key features responsible for its lubrication $^{5}$. One is that the tissue is a fluid-filled porous matrix in which most of the compressive load is carried by the fluid itself, as it is trapped and pressurized. This reduces the stress on the matrix, but it doesn't alone account for lubrication and wear resistance. Furthermore, highly charged macromolecules called proteoglycans bound to the surface both resist collapse of the pores through electrosteric repulsion and provide detachable 'boundary lubricants' under shear.

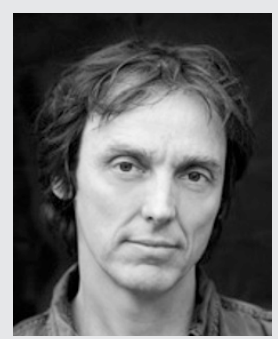

PHILIP BALL

In the analogue material developed by Greene et al., cellulose with submicrometre porosity mimics the collagen fibril network of cartilage, while the boundary lubrication is supplied by copolymers of the polyelectrolyte carboxymethylcellulose with polyethylene glycol. The resulting material shows not only low friction (albeit higher than real cartilage) but also the time-dependent static frictional response diagnostic of the fluid-pressurization mechanism of lubrication. This composite isn't yet robust enough for medical applications, but it shows that the strategy is sound.

References

1. Natoli, R. M., Revell, C. M. \& Athanasiou, K. A. Tissue Eng. A 15, 3119-3128 (2009).

2. McNary, S. M., Athanasiou, K. A. \& Reddi, A. H. Tissue Eng. B 18, 88-100 (2012).

3. Mow, V. C., Ratcliffe, A. \& Poole, A. R. Biomaterials 13, 67-97 (1992).

4. Chen, M., Briscoe, W. H., Armes, S. P. \& Klein, J. Science 323, 1698-1701 (2009).

5. Greene, G. W., Olszewska, A., Osterberg, M., Zhu, H. \& Horn, R. Soft Matter http://dx.doi.org/10.1039/ c3sm52106k (2013).

\section{Nanoscale oxides shape up}

Reversible strains up to $14 \%$ driven by changes in temperature or electric field have been realized in a thin film of bismuth ferrite oxide.

\section{Antoni Planes and Lluís Mañosa}

B end a paper clip, and its shape will persist even if the clip is heated up. This is because the deformed clip has undergone irreversible plastic deformation through the flow of dislocations. This type of crystallographic defect - which can be viewed as misfit lines between crystallographic planes - confers ductility to metals because they can easily move under an applied load. Yet, in contrast to most metals, shape-memory alloys respond very differently to applied loads. In this case, the response of the material is not controlled by dislocation flow, but by a transition between two crystallographic phases, a low-symmetry martensitic phase and a high-symmetry parent phase ${ }^{1}$. At the transition, and in the absence of external 